Copyright (C1996, American Institute of Aeronautics and Astronautics, Inc.

AIAA Meeting Papers on Disc, July 1996

A9637364, AIAA Paper 96-3298

\title{
Plume characterization of the SPT-100
}

\author{
Alec D. Gallimore \\ Michigan Univ., Ann Arbor \\ Brian E. Gilchrist \\ Michigan Univ., Ann Arbor \\ Lyon B. King \\ Michigan Univ., Ann Arbor \\ Shawn G. Ohler \\ Michigan Univ., Ann Arbor
}

\author{
Alranzo B. Ruffin \\ Michigan Univ., Ann Arbor
}

\section{AIAA, ASME, SAE, and ASEE, Joint Propulsion Conference and Exhibit, 32nd, Lake Buena Vista, FL, July 1-3, 1996}

\begin{abstract}
A first-year summary is presented of a research program sponsored by the USAF to characterize the SPT-100 for far-field plume plasma and transport properties, as well as thruster operation impact to EM signals used for communication, radar, and navigation systems. An extensive array of electrostatic and flux probes is used to assess particle transport within the thurster's plume; a new Neutral Particle Flux Sensor has been developed and used. One of the most striking results is the discovery of a high energy annulus surrounding a low energy core in the plume at $0.5 \mathrm{~m}$ radius from the thruster exit, which retains certain characteristics out to at least $1 \mathrm{~m}$. Charge exchange products have also been measured which help quantify energetic neutral return flux. EM signal characterization has been able to quantify significant signal attenuation through out the thruster plume for low microwave signals near the local plasma frequency. It has also been possible to demonstrate that noise from the plume is induced into the EM signal by both amplitude and phase modulation processes. (Author)
\end{abstract}




\title{
PLUME CHARACTERIZATION OF THE SPT-100
}

\author{
Alec D. Gallimore $\S^{\S}$, Brian E. Gilchrist $\$ \ddagger$, \\ Lyon B. Kingl, Shawn G. Ohler ${ }^{*}$, and Alranzo B. Ruffin ${ }^{\dagger}$ \\ University of Michigan \\ Ann Arbor, MI
}

\begin{abstract}
The following provides a first year summary of a research program sponsored by the AFOSR to characterize the SPT- 100 for far-ficld plume plasma and transport properties as well as thruster operation impact to electromagnetic signals used for communication, radar, and navigation systems. An extensive array of electrostatic and flux probes are being used to assess particle transport within the thurster's plume. This includes a Retarding Potential Analyzer, a Faraday Probe and Convective Heat Flux Probe. In addition, a new Neutral Particle Flux (NPF) Sensor has been developed and used. A high-fidelity Molccular Beam Mass Spectrometer is under development as well as a new insitu probe to characterize flow velocity, electron temperature, and cstimate ion temperature within an order of magnitude using the characteristics of an ion acoustic wave. One of the most striking results of this investigation to date has been the discovery of a high energy annulus surrounding a low energy core in the plume at $0.5 \mathrm{~m}$ radius from the thruster exit which retains certain characteristics out to at least $1 \mathrm{~m}$. Charge exchange products have also been measured which help quantify energetic neutral return flux. Electromagnetic signal characterization has been able to quantify significant signal attenuation through out the thruster plume for low microwave signals near the local plasma frequency. It has also been possible to demonstrate that noise from the plume is induced into the electromagnetic signal by both amplitude and phase modulation processes.
\end{abstract}

\section{Introduction}

In an effort to fully flight-qualify the SPT- 100 for use on communication satellites, a great deal of research has focused on lifetime and performance issues of the thruster. As a result, the baseline opcrating conditions of the SPT-100 are now well established. The next hurtle to widespread use of these devices is an understanding of thruster/spacecraft interaction phenomena. The thruster may interact with the spacecraft through four fundamental processes: thruster-generated clcctromagnetic interference (EMI), particle impingement from the exhaust plume, radiative heating of spacecraft components, and the adverse interaction of radio frequency (RF) signals with the plume plasma. EMI may interfere with spacceraft components and may degrade instrument performance. Particle impingement and radiant heating may causc physical damage to spacecraft hardware, while the plume plasma may degrade spacecraft communication signal quality.

A research program, sponsored by the Air Force Office of Science and Research (AFOSR) has therefore been organized to accomplish two principal activities: (i) a detailed survey of far-field plume

\footnotetext{
$\$$ Assistant Professor, Aerospace Enginecring, member AIAA

\# Assistant Professor, Electrical Engineering and Computer Science, member AIAA

I Graduate Student Research Assistant, Aerospace Engineering. menther AIAA

* Graduate Student Research Assistant. Electrical Enginecring and Computer Science, member AIAA

$\dagger$ Graduate Student Restarch Assistant, Applied Physics Department
}

plasma and transport properties; and (ii) a detailed assessment of the impact thruster operation may have on spacecraft electromagnetic signals such as used for communication, radar, and navigation systems. At the time of this writing, the research program has been underway for approximately one year. However, considerable progress in both research areas has already been made and is summarized here. We also note that the results of this research is supporting Particle-in-Cell (PIC) code validation at the Massachusetts Institute of Technology as well as spacecraft systems studies at Space Systems/Loral and NASA Lewis Research Center.

All measurements reported here were performed in the Plasmadynamics and Electric Propulsion Laboratory (PEPL) at the University of Michigan. The centerpicce of this laboratory is a large $6 \mathrm{~m}$ dia. by $9 \mathrm{~m}$ long space simulation vacuum chamber capable of base pressures near $2 \times 10^{-5}$ Torr. A detailcd description of this facility has been published elsewhere. ${ }^{1}$ Measurements were made of a Fakel Enterprises SPT-100 flight model thruster (Figure 1), on loan from Space System/Loral, and powered by a Space Systems/Loral Power Processing Unit (PPU).

Below, Section 2 describes Far-field plume particle impact studies. Section 3 describes recent results from communications impact studies and modeling. Section 4 summarizes our progress and discusses plans for the future. 


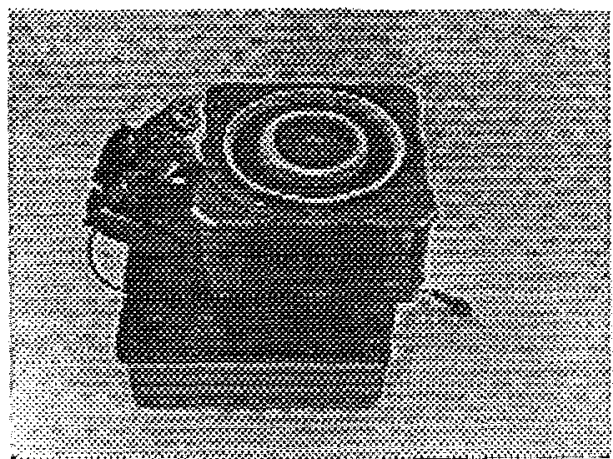

Figure 1. Stationary Plasma Thruster, SPT-100.

\section{2• Highlights of Plume Particle Studies}

Under the AFOSR sponsored research program at the University of Michigan, the exhaust plume particle-based properties are being quantified using a combination of proven electrostatic probe techniques along with some new diagnostic techniques developed specifically for this research. The chronological order of these interaction studies are following a "top down" approach. Initially, spatial maps of total and radiant heat flux as well as ion current density have been measured over an extensive volume of the plume. $^{2}$ These data can be immediately used to aid in the design of thermally sensitive components as well as the cstimate of plume-induced spacecraft charging.

Secondly, a more extensive array of electrostatic probes including a Retarding Potential Analyzer (RPA) are being used to provide greater insight into particle transport properties within the plume. From a gaskinetic standpoint, the RPA measures the ion energy distribution function, while a Faraday Probc and a Convective Heat Flux Probe measure the first and second moments of the distribution function, respectively. These measurements can provide ion density as well as ion velocity. In addition, a new Neutral Particle Flux (NPF) sensor is being developed. ${ }^{3}$ The NPF utilizes an array of electrostatic grids to repel the charged particle flux while a hot cathode ionization gauge located downstream of the grids acts as a detector for neutral particle flux. These data can be used to gain insight into propellant ionization as well as facility-induced charge exchangc collisions (CEX) with the ambient gas.

Construction of a high-fidelity Molecular Beam Mass Spectrometer (MBMS) has been initiated. The MBMS will consist of a differentially pumped ultrahigh vacuum sub-chamber which will sample a portion of the exhaust plume through a small orifice skimmer. The resulting beam will be electrostatically filtered to allow for the measurement of both species concentration and kinetic cnergy. For example, this instrument should allow for the relative spatial concentration and absolute energy distribution of thruster erosion products to be measured in realtime. This sensor should be operation by the summer of 1997.

Lastly, a diagnostic technique is being developed to simultaneously characterize flow velocity, elcctron temperature, and estimatc ion temperature to within an order of magnitude. The technique uses the characteristics of an ion acoustic wave that convectively propagates along the flowing plasma in the plume. This technique is relatively independent of the probe-plasma coupling and produces minimal disturbance in the plasma.

Combining knowledge gaincd from measurements using these probes, it has been possible to quantify the transport of mass, energy, and charge within the plume at $0.5 \mathrm{~m}$ and $1.0 \mathrm{~m}$ radius from thruster exit. Below, we highlight some of these recent measurements.

Distribution of Ion Energy. As reported by King and Gallimorc ${ }^{2}$, one of the most striking results of this investigation to date has been the discovery of a high energy annulus surrounding a low energy core in the plume at $0.5 \mathrm{~m}$ radius from thruster exit (Figure 2). Although such a topology would be expected in the near field of the thruster due to the annular shape of the discharge chamber, it was believed that the divergence of the ion beam would Iill in this configuration very rapidly. On the contrary, the average ion centerline velocity at $0.5 \mathrm{~m}$ is less than the velocity computed for any other point measured in the $0.5 \mathrm{~m}$ data set. This low energy core is very narrow, limited to a region in space with a half angle of 5 deg. It should be noted, however, that this annulus is not manifested by a corresponding trend in ion current density. The ion density increases toward centerline at a much larger rate than the ion velocity shift; consequently plasma density measurements as well as current density measurements fail to uncover this annulus/core configuration. 4

The average ion velocity data at $1.0 \mathrm{~m}$ showed a similar trend. Although the low encrgy core has been essentially filled in by the diverging ion beam, the high energy annulus is still apparent at a slightly greater angle off centerline.

Charge Exchange Processes. The products of charge exchange (CEX) collisions can be determined in two ways: one can either look for the fast neutrals or the corresponding slow ions, since their production rates are one-to-one. Figure 3 shows the NPF probe based calculations of CEX neutral particle velocity and density at $0.5 \mathrm{~m}$ radius from thruster. 


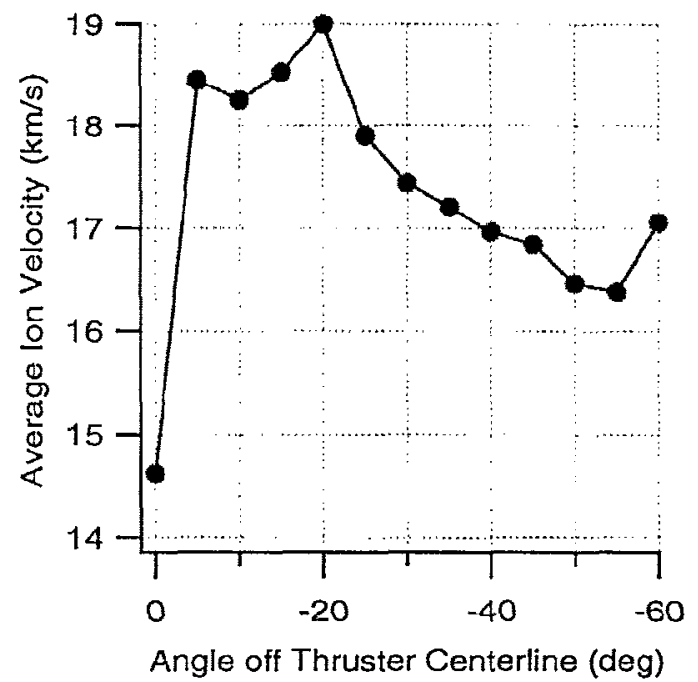

Eigurc 2. Average ion velocity as computed from the first moment of the RPA-derived distribution function at $0.5 \mathrm{~m}$ radius from thruster exit.

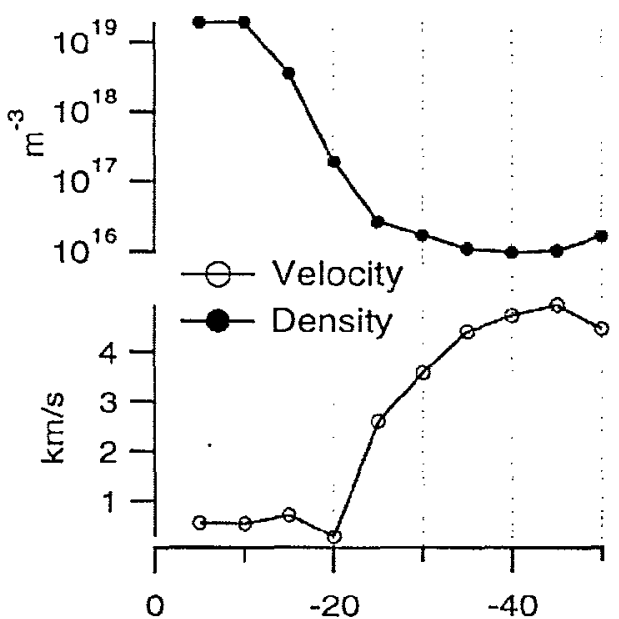

Angle off Thruster Centerline (deg)

Figure 3. Fast CEX neutral particle density and velocity at 0.5 radius from thruster exit plane.

The slow-ion products of CEX were measured by turning the Faraday probe "backward" in the plume flow. In this configuration the probe body shielded the collector from the direct flux of plume ions and created a quiet wake. By sampling plasma properties in this wake the current density of slow CEX ions were measured. Figure 4 shows both the direct plume flux and the slow wake flux due to the CEX ions.

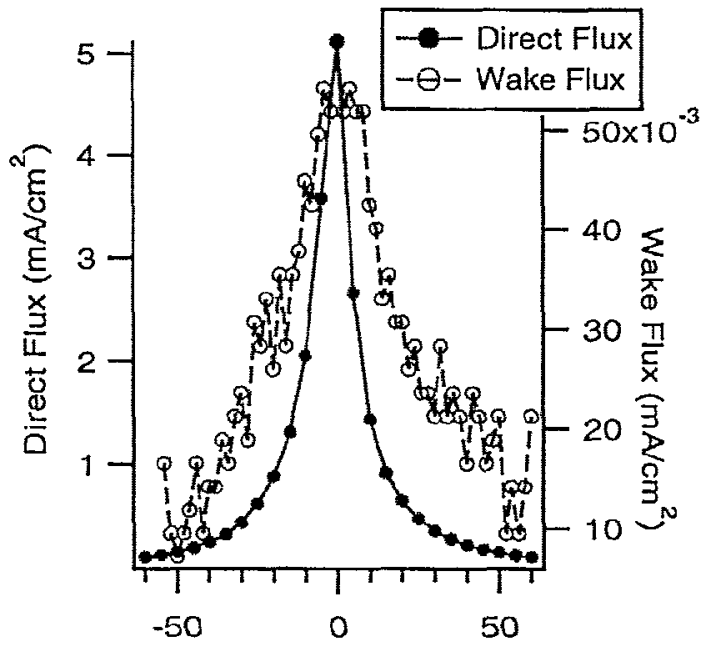

Angle off Thruster Centerline (deg)

Eigure 4. Faraday probe-measured ion current density at $0.5 \mathrm{~m}$ radius from thruster exit. Data is shown for both direct plume impingement and for ambient wake current density.

Heat Flux Probe. The heat flux probe was used to map out the thermal environment within the thruster plume. Both total (convective plus radiant) and radiant heat flux were measured at $0.5 \mathrm{~m}$ and $1.0 \mathrm{~m}$ from thruster exit. The $0.5 \mathrm{~m}$ data is shown as Figure 5.

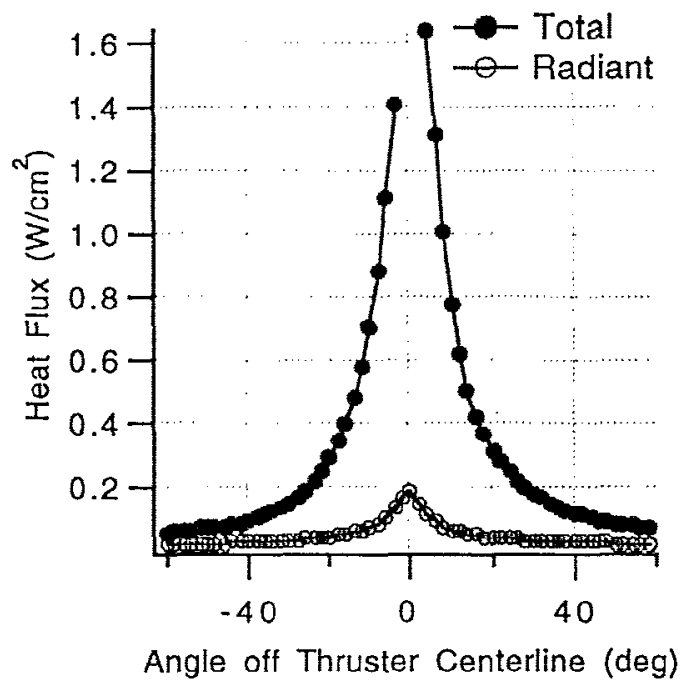

Figure 5. Total and Radiant heat flux at $0.5 \mathrm{~m}$ radius from thruster exit plane.

The explanation for the observations is discussed in detail by King and Gallimore ${ }^{2}$, where it is noted that the existence of a high energy plasma annulus with low spatial divergence is consistent with the thruster discharge chamber geometry and field 
topology. The higher energy ions are formed further back inside the discharge chamber. These ions, in order to escape the discharge chamber without colliding with the wall, must have their velocity vectors contained within a defined solid angle. Those ions formed near the end of the discharge chamber will have lower energy as a consequence of the field topology, and will also have their velocity vectors distributed about a larger solid angle. Such a distribution of velocity vectors would produce a depletion of high energy ions on thruster centcrline until a considerable distance downstream. Such a scenario is depicted in Figure 6.

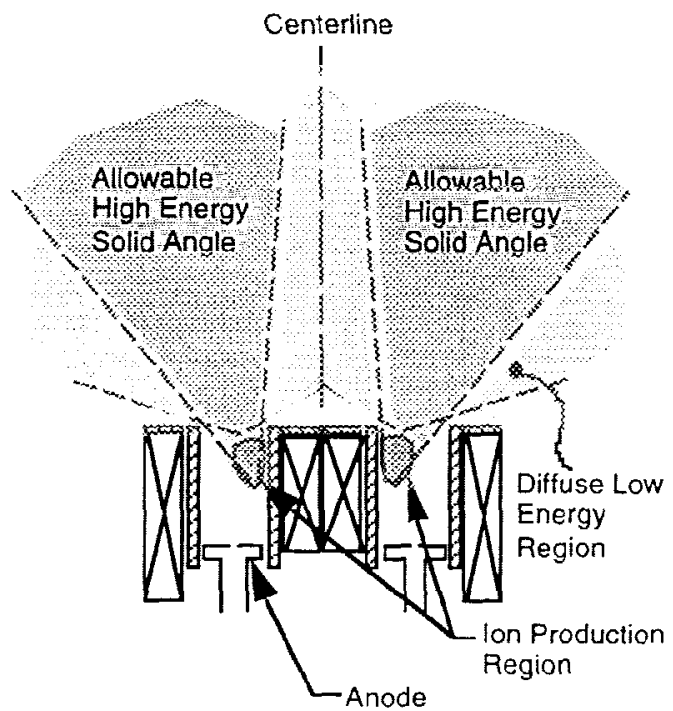

Figure 6. Schematic representation of mechanism causing high encrgy annulus and low energy core in thruster plume. Ion production region denoted as according to Bishaev and Kim. ${ }^{5}$ High energy ions are formed closest to anode.

\section{3*Highlights of Electromagnetic Signal Impact Studies}

The SPT-100 thruster plume generates plasma densities with corresponding plasma frequencies approaching $3 \mathrm{GHz}$ and measurable effects to elcctromagnetic signals well above this frequency are to be expected for electromagnetic signals passing through the plume. ${ }^{4}$ In particular, the plume potentially degrades the electromagnetic signal through attenuation as well as added amplitude or phase noise. Thus, consideration must be given to understanding the extent and kinds of interactions with satellite elcctromagnetic systems. This is bcing accomplished through a combination of experimental measurements and theoretical propagation models as described by Ohler et al. ${ }^{6}$

Building upon our previous results ${ }^{4}$ with $\mathrm{Ku}-$ band signals $(17 \mathrm{GHz})$, laboratory experiments were recently performed to characterize the impact that the SPT-100 plume has on L-band $(1.575 \mathrm{GHz})$ communications signals in terms of phase shift, phase noise, and attenuation. In addition, more complete ray-tracing models are being developed to quantify attenuation and amplitude or phase noise modulation

Simulation of Attenuation and Amplitude Modulation. Ray tracing is an extremely useful tool lor predicting high frequency electromagnetic transmission characteristics. This work utilizes the ray tracing technique to simulate the attenuation and amplitude modulation of a signal transmitted through an SPT plume. Ray tracing separates the components of the electromagnetic fields so that the amplitude, phase, and ray path are traced individually. This work focuses on finding the power density of the ray and characterizing the change in power density over time.

Using the ray tracing analysis technique and an assumption of power conservation, it is possible to model the amplitude, phase, and ray path as it propagates through the plume. An example of one possible propagation path is shown on the left side of Figure 7 while the right side shows the resulting defocusing effects (refraction) due to the plasma plume and its spatial variations.

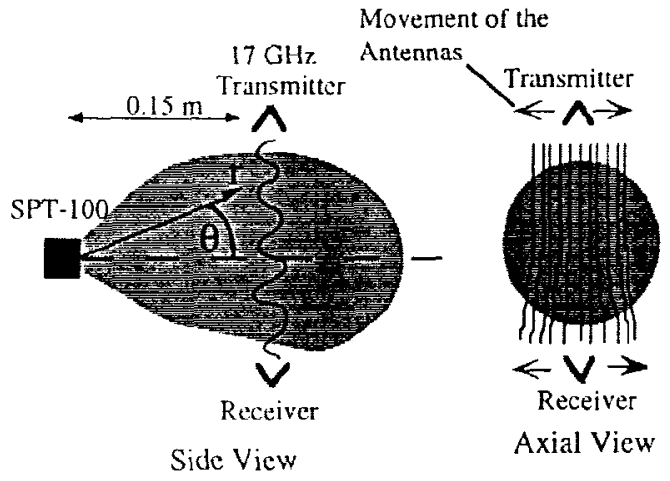

Figure 7. Physical System for Ray Trace Modeling.

A series of rays are used to simulate an antenna aperture through the superposition of the electric fields across the aperture. The amplitude of the individual rays, the simulated antenna power, and measurement results at similar conditions are plotted in Figure 8. The plots show the expected relationship between the individual rays and the antenna; the antenna results are the convolution of the individual rays and the antenna function. The differences between measurement and simulation can be attributed to the inherent limitations of the ray tracing technique. Two simulation limitations are the finite ray sampling and step size both having greater importance at smaller wavelengths where the error tends 10 affect the phase more than the amplitude. 
Two other limitations are the electron density model which is thought to be accurate only to $\pm 20 \% 4$ and the limited accuracy of the antenna distribution function. Given these factors, the difference between the ray tracing simulations and the experimental measurements is expected.

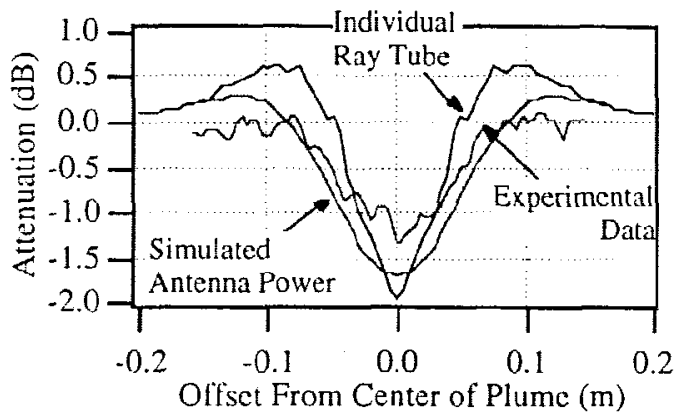

Figure 8. Power Change Due to Plasma for Individual Ray Tubes, Simulated Antenna, and Experimental Results; $0.15 \mathrm{~m}$ downstream, $17 \mathrm{GHz}$.

The power spectral density is a useful description for determining the quality of an electromagnetic signal. The simulated power spectral density is found for a $17 \mathrm{GHz}$ signal transmitted through the plume $0.15 \mathrm{~m}$ from the exit plane using modulation parameters from the ray tracing simulation (Figure 9 a).

The plasma oscillations generate $26 \mathrm{kHz}$ harmonics where the first harmonic is produced through a combination of the amplitude and phase modulation. The first harmonic compares well (within $2 \mathrm{~dB}$ ) with the measured results in Figure 9b; however, the simulation under-predicts the measured sccond harmonic. In addition, the broad band noise exhibited in the measurements are not predicted by the single frequency oscillation model. The differences between simulations and experiments would decrease with a more accurate model of the spectral components of the plume oscillations.

In summary, density oscillations in the plume produce both amplitude and phase modulation. This evaluation demonstrated the mechanism of amplitude modulation by the plume through ray tracing simulations. Additionally, the simulations compared well with measurements for a similar expcrimental siluation.

Signal Characterization at $1.575 \mathrm{GHz}$. Experimental characterization in the low microwave frequency regime is important because ray tracing simulations near or bclow the plasma frequency are not possible. Therefore, an L-band (1.12-1.7 GHz) microwave measurement system (Figure 10) has been employed to measure the altenuation, phase shili, spcctral power distribution, and phase noise of a $1.575 \mathrm{GHz}$ signal (GPS frequency) through the xenon plasma exhaust of an SPT- 100 thruster 6

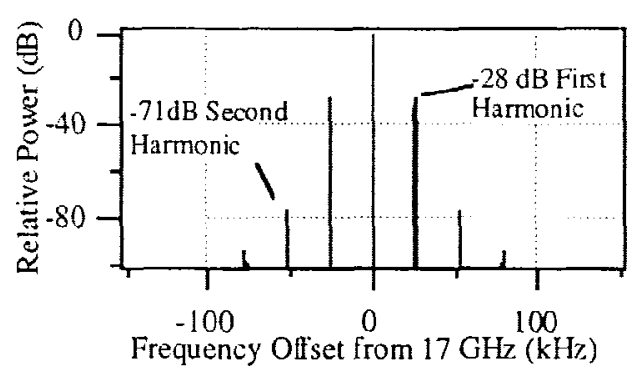

(9a) Simulated Effect of Density Oscillations

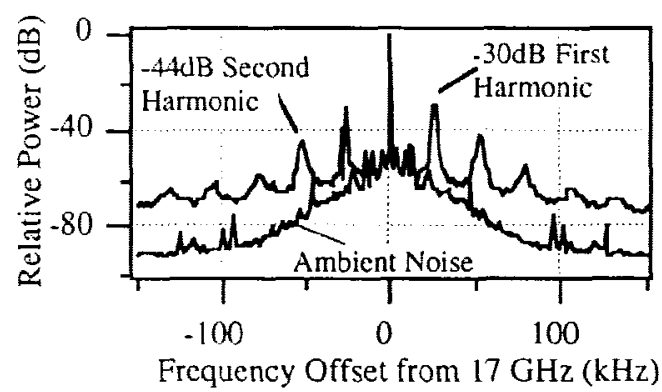

(9b) Measured Power Spectral Density

Figure 9. Comparison of the Simulated and Measured Power Spectral Density of a $17 \mathrm{GHz}$ Signal Transmitted Across the Plume $0.15 \mathrm{~m}$ from the Exit Plane.

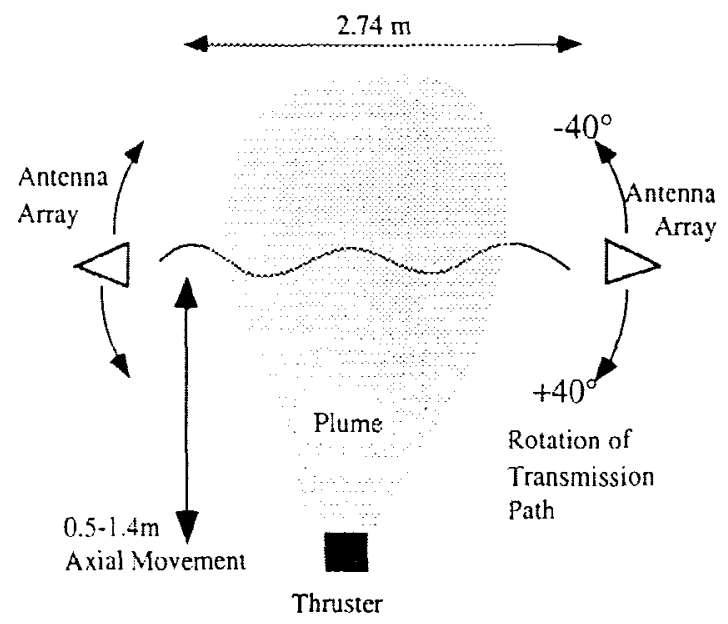

Figure 10. Top View of L-band Antenna Experiment.

In this experiment, we measure the attenuation, phase shift, and power spectral density of the signal at axial distances from the exit plane of 0.5 to $1.4 \mathrm{~m}$, with angular positions set at $0^{\circ}, 10^{\circ}, 20^{\circ}, 30^{\circ}$, and $40^{\circ}$ relative to the position shown in Figure 10. The sizable attenuation effects are shown in Figure 11 as an example 


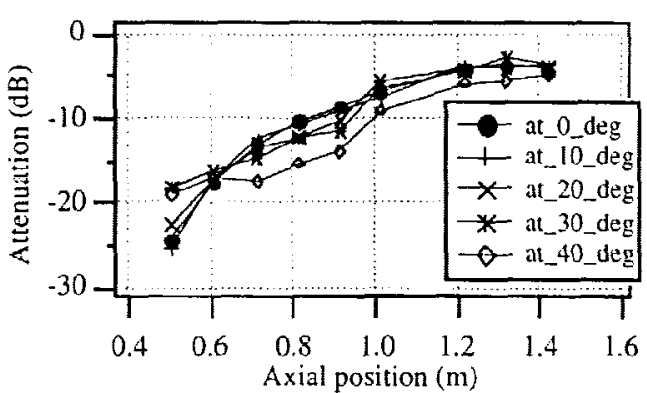

Figure 11. Attenuation Levels for 1.6 GHz Transmission Across the Plume for Various Axial Positions and Transmission Angles.

Power spectral density information has also been obtained over the same position set as the amplitude and phase. A representative measurement is contrasted to measurements at $17 \mathrm{GHz}$ and a baseline ambient measurement (Figure 12). As expected, the lower frequency couples into the plasma much more strongly than the $17 \mathrm{GHz}$ signal. The $1.575 \mathrm{GHz}$ signal has as much as $30 \mathrm{~dB}$ more noise power than the $17 \mathrm{GHz}$ system and as much as $40 \mathrm{~dB}$ higher than the noise floor of the microwave system without the plasma present. As with the $17 \mathrm{GHz}$ system, the most significant coupling occurs at the dominant plasma oscillation frequency, $26 \mathrm{kHz}$.

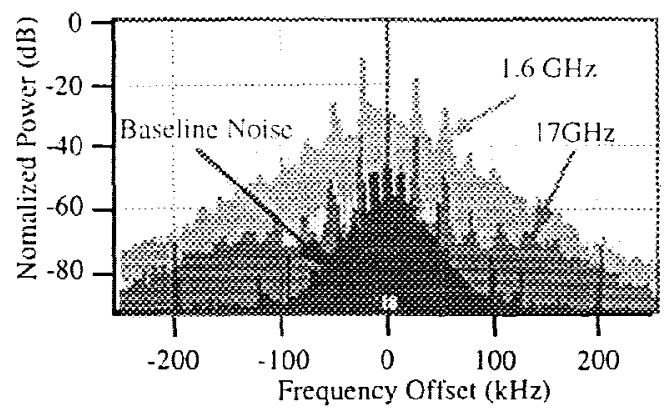

Figure 12. Comparison of Power Spectral Density of Signal Transmitted at $0.5 \mathrm{~m}$ for $1.6 \mathrm{GHz}, 17 \mathrm{GHz}$, and a Common Baseline without a Plume.

Further simulations for a wide range of conditions indicate trends in the amplitude and phase over a range of frequencies and axial positions. The attenuation indicates a sharp increase in power loss at the lower frequencies, but also a quick reduction in plume impact with increasing distance. The modulation coefficient follows similar trends indicating up to $25 \%$ modulation at the closest measurement point at $3 \mathrm{GHz}$ and also indicating less than $5 \%$ modulation for all distances at $17 \mathrm{GHz}$; however, as was demonstrated earlier with the $17 \mathrm{GHz}$. simulation, even small modulation cocfficients produce significant increases in the noise power.
Representative simulation results are plottcd in Figure 13 where experimental data at $1.575 \mathrm{GHz}$ and $17 \mathrm{GHz}$ correlates well with the simulations.

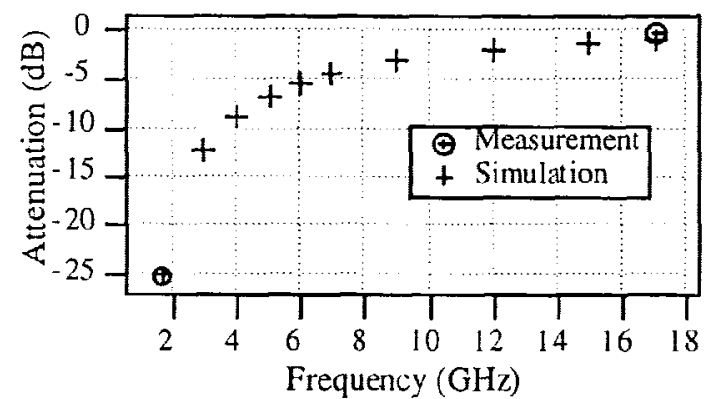

Figure 13 . Representative ray tracing attenuation simulations with comparable measurements at 1.575 $\mathrm{GHz}$ and $17 \mathrm{GHz}$.

For any given position the failure point for the ray tracing simulation is along the thruster axis since it is the location for highest plasma density. The contour defining the limiting frequency is plotted in Figure 14. Using this condition as a boundary, the attenuation below the limit is expected to be greater than $10 \mathrm{~dB}$. This conclusion is drawn from the experimental results at $1.575 \mathrm{GHz}$ and extrapolation of the ray tracing simulations.

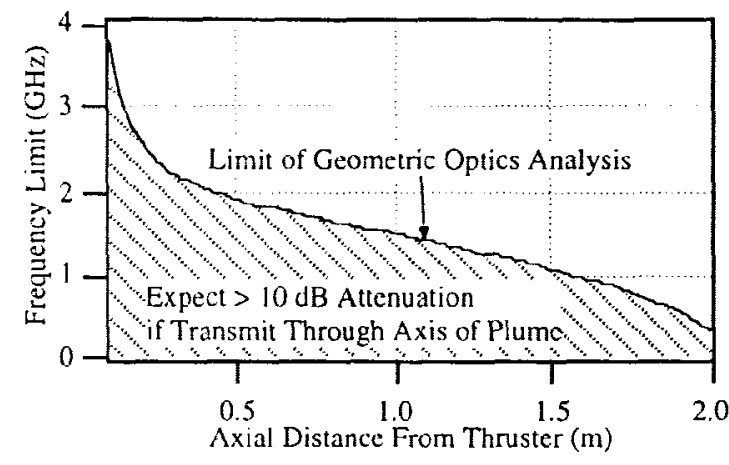

Figure 14. Theoretical Frequency Limit of Geometric Optics With Respect to Transmitting Across the Plume at a Given Axial Distance From the Thruster Exit Plane.

\section{Summary}

The above has been a summary of progress over the first year of a two pronged research program to characterize the SPT-100. Greater detail of these results are given by King and Gallimore 2,3 for the survey of far-field plume plasma and transport properties and by Ohier et al. ${ }^{6}$ for thruster operation impact to electromagnetic signals. In the coming ycar, more detailcd measurements are expected of the plasma transport properties of the SPT-100, D-55, and D-100 plumes using the array of diagnostics presented in this paper. Furthermore, the new MBMS should be operational by the summer of 
1997. For the electromagnetic signal characterization, a survey of model predictions will be completed to quantify the expected effects both spatially and over frequency. Impact to frequencies higher than $20 \mathrm{GHz}$ are being considered as well as a survey of noise response as a function of thruster operating conditions.

\section{References}

[1] Gallimorc, A.D., S.-W. Kim, J. E. Foster, L. B. King, F. S. Gulczinski III, "Near- and Far Ficld Plume Studies of a Onc-Kilowatt Arcjet," AIAA Journal of Propulsion and Power, Vol 12, No. 1, Jan.-Feb., 1996, pp 105-111.

[2] King, L.B., and A.D. Gallimore, "Tonic and Neutral Particle Transport Property Measurements in the Plume of an SPT100," AIAA-96-2712, Joint Propulsion Conference, July 1-3, 1996, Orlando, FL.

[3] King, L.B., and Gallimore, A.D., "A Gridded Retarding Pressure Sensor for Ion and Neutral Particle Analysis in Flowing Plasmas," AIAA-96-2983, Joint Propulsion Conference, July 1-3, 1996, Orlando, FL.

[4] Ohler, S. G, B. E. Gilchrist, and A. D. Gallimore, "Microwave plume measurcments of an SPT-100 using Xenon and a laboratory model SPT using Krypton," Joint Propulsion Conference, San Diego, CA, Junc, 1995.

[5] Bishaev, A.M., and Kim, V., "Local Plasma Propertics n a Hall-current Accelerator with an Extended Acceleration Zone," Sov. Phys. Tech. Phys. 23(9), Sept. 1978, pp. 1055-57.

[6] Ohler, S. G, A. B. Ruffin, B. E. Gilchrist, and A. D. Gallimore, "RF Signal Impact Study of an SPT," AIAA-96-2706, Joint Propulsion Conference, July 1-3, 1996 , Orlando, FL. 